\title{
Neue Patientenbroschüre zu chronischen Zystitiden
}

— Eine Schädigung der Glykosaminoglykan-(GAG)-Schicht gilt als wahrscheinliche Ursache für die Entstehung chronischer Zystitiden. Eine seit Jahren etablierte, kausalnahe und wissenschaftlich breit dokumentierte Behandlungsmöglichkeit bei chronischen Zystitiden ist die GAG-Ersatztherapie mit 0,2\%igem Chondroitinsulfat (Gepan ${ }^{\circledR}$ instill).

Die wichtigsten Fragen Ihrer Patienten zur GAG-Ersatztherapie beantwortet der Patientenratgeber von Gepan ${ }^{\circledR}$ instill „Die Lösung bei chronischen Blasenentzündungen“. Er informiert anschaulich über die verschiedenen Krankheitsbilder und Symptome bei chronischen Zystitiden und erklärt die Behandlung mit Gepan ${ }^{\circledast}$ instill. Damit unter- stützt der Ratgeber das Patientengespräch in der Praxis.

Interstitielle Zystitis, überaktive Blase und radiogene Zystitis - den Symptomen dieser Zystitiden liegt häufig ein Defekt der GAGSchicht zugrunde. Mit ihrem wesentlichen Bestandteil Chondroitinsulfat bildet diese Blasenwandschutzschicht die Grenze zwischen dem Urin und dem Urothel. Ist die GAG-Schicht geschädigt und damit die physiologische Schutzfunktion gestört, können reizende Substanzen aus dem Urin bis in die tiefer gelegene Schichten der Blasenwand vordringen. Dies löst die typischen Symptome chronischer Zystitiden wie Harndrang, erhöhte Miktionsfrequenz oder Schmerzen in der Beckenregion aus. Die intravesikale
Instillation mit 0,2\%igem Chondroitinsulfat versorgt die GAG-Schicht mit Chondroinsulfat und behebt den Defekt der Blasenschutzschicht. Gepan ${ }^{\circledast}$ instill ist das einzige Präparat, das als Fertigspritze zur Verfügung steht. Es ist einfach und schnell zu applizieren und Kontaminationen werden verhindert. Wissenschaftliche Studien belegen, dass sich die klinischen Symptome unter der GAGErsatztherapie verbessern.

Die Patientenbroschüre von Gepan ${ }^{\circledR}$ instill können Ärzte per E-Mail bestellen (info@ pohl-boskamp.de).

Nach Informationen von

Pohl-Boskamp, Hohenlockstedt

\section{BPH: Kombinationstherapie deutlich wirksamer als Monotherapien}

— Fast $50 \%$ der Männer ab 50 Jahren leiden unter einer benignen Prostatahyperplasie (BPH). „Schon bei einer moderaten Erkrankung ist die Lebensqualität der Patienten deutlich beeinträchtigt ", betonte Dr. Volker Moll, Augsburg, und fügte hinzu:,Indem man die Beschwerden behandelt, kann man im gleichen Maße auch die Lebensqualität verbessern."

Die Wirksamkeit einer Kombinationstherapie im Vergleich zu den jeweiligen Monotherapien wurde in einer aktuellen Studie untersucht [Roehrborn CG et al. Eur Urol. 2010;57(1):123-31]. Im Rahmen der randomisierten, doppelblinden CombAT (Combination of Avodart ${ }^{\circledR}$ und Tamsulosin)-Studie wurden 4.844 mindestens 50-jährige Männer mit moderaten bis schweren BPH-Symptomen behandelt. Die Symptome wurden durch den IPSS (International Prostate Symptom Score) gemessen. Die Patienten erhielten 0,5 mg Dutasterid (Avodart ${ }^{\circledR}$ ), 0,4 mg Tamsulosin oder die Kombination aus beiden Medikamenten einmal täglich

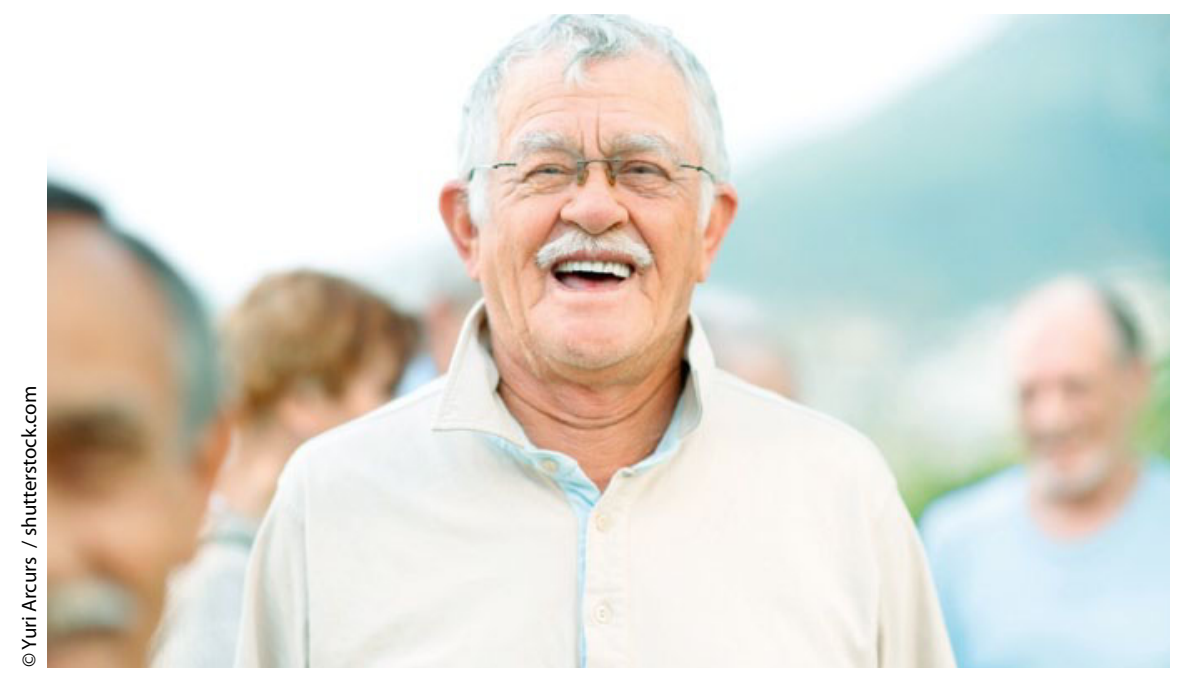

Mit einer Kombinationstherapie lassen sich die BPH-Symptome signifikant besser lindern als mit einer Monotherapie.

Im Beobachtungszeitraum von vier Jahren sank das relative Risiko eines akuten Harnverhalts oder einer BPH-bedingten Operation unter der Kombinationstherapie im Vergleich zur Monotherapie mit Tamsulosin signifikant um $65,8 \%(p<0,001)$ und um $19,6 \%$ im Vergleich zur Monotherapie mit Dutasterid $(p=0,18)$.

Das Risiko einer klinischen Progression der BPH war bei kombinierter Gabe gegenüber Tamsulosin-Monotherapie um 44,1\% und gegenüber der alleinigen Behandlung mit Dutasterid um $31,2 \%$ reduziert.

„Die Kombination hat die größere Symptomlinderung hervorgerufen. Nach drei Monaten war sie der Monotherapie mit Dutasterid und nach neun Monaten der Monotherapie mit Tamsulosin überlegen, $p<0,001$. Diese Symptomlinderung hielt über den gesamten Studienzeitraum von vier Jahren an", erläuterte PD Dr. Christian Gratzke, München. Die Verträglichkeit sei sehr gut gewesen, es seien keine neuen überraschenden Nebenwirkungen aufgetreten, so Gratzke. Seit Juni 2010 gibt es die Kapsel Duodart ${ }^{\oplus}$, die beide Wirkstoffe enhält.

Kamingespräch „Benigne Prostatahyperplasie - Chancen einer neuen Behandlungsoption", München, 23. Februar 2011

Veranstalter: GlaxoSmithKline, München 